\title{
Intestinal inflammation affected by density of enteric neurons
}

The density of enteric neurons affects the severity of intestinal inflammation in mouse models of colitis and could be linked to the pathogenesis of IBD, according to the results of a new study published in Gastroenterology. "The density of enteric innervation may be a factor that is directly related to predisposition to intestinal inflammatory disease," explains first author Kara Margolis.

The gastrointestinal tract is controlled by the enteric nervous system (ENS), which helps regulate motility, secretion and immunity in the gut. Existing evidence has shown that enteric neurons are increased in areas of intestinal inflammation in patients with IBD. Exactly how the ENS is linked with intestinal inflammation is, however, unclear. "Other scientists have speculated that this hyperinnervation was a result of inflammation," says Margolis. "It seemed reasonable that the hyperinnervation could actually be a precipitant or factor that increased the severity of inflammation."

In their study, Margolis and colleagues used transgenic mice with altered numbers of enteric neurons: one with an increased number of enteric neurons (hyperinnervated, NSE-noggin mice) and one with fewer enteric neurons (hypoinnervated, Hand $2^{+/-}$mice). They then induced colitis in these mice using either trinitrobenzene sulfonic acid (effects similar to Crohn's disease) or dextran sulfate sodium (effects similar to ulcerative colitis) and measured the levels of intestinal inflammation by examining a number of factors, including colitis symptoms and expression of proinflammatory genes and proteins.

The observed level of inflammation in both types of colitis was determined by the number of enteric neurons; hyperinnervated mice had increased inflammation, whilst hypoinnervated mice had decreased inflammation compared with wild-type mice. Importantly, the
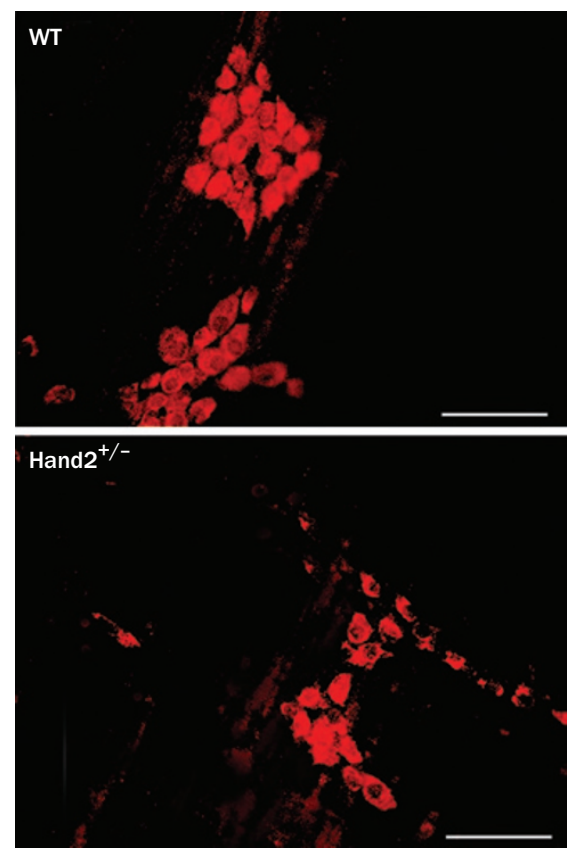

Samples of the myenteric plexus from wild-type (WT) and Hand $2^{+-}$mice stained with HuD, a marker of neuronal cell bodies. Enteric neurons in Hand2 ${ }^{+-}$mice are smaller and decreased in number compared with WT mice. Scale $=40 \mu \mathrm{m}$. Courtesy of K. Margolis.

differences between the inflammatory responses in the transgenic mice were confirmed to be specific to the gut and not because of an overall altered immune response (the systemic inflammatory response was similar to wild-type mice in both mutant mice).

Margolis believes the findings could help to "push forward scientific advancement in the field of neuroimmune interactions in intestinal inflammatory diseases". She concludes that "a preexisting hyperinnervation of the intestine, or segments of the intestine, may cause or predispose people to intestinal inflammation or may worsen inflammation once it occurs".

\section{Katrina Ray}

Original article Margolis, K. G. et al. Enteric neurona density contributes to the severity of intestinal inflammation. Gastroenterology 141, 588-598 (2011) 